CLINICAL STUDY

\title{
Impact of metformin versus repaglinide on non-glycaemic cardiovascular risk markers related to inflammation and endothelial dysfunction in non-obese patients with type 2 diabetes
}

\author{
Søren S Lund ${ }^{1}$, Lise Tarnow ${ }^{1}$, Coen D A Stehouwer ${ }^{2}$, Casper G Schalkwijk ${ }^{2,3}$, Tom Teerlink ${ }^{3}$, Jørgen Gram ${ }^{4}$, \\ Kaj Winther ${ }^{5}$, Merete Frandsen ${ }^{1}$, Ulla M Smidt ${ }^{1}$, Oluf Pedersen ${ }^{1,6}$, Hans-Henrik Parving ${ }^{6,7}$ and Allan A Vaag ${ }^{1,8}$ \\ ${ }^{1}$ Steno Diabetes Center, Niels Steensens Vej 2, 2820 Gentofte, Denmark, ${ }^{2}$ Division of General Internal Medicine, Department of Internal Medicine, \\ University Hospital Maastricht, 6202 AZ Maastricht, The Netherlands, ${ }^{3}$ Department of Clinical Chemistry, VU University Medical Centre, 1007 MB \\ Amsterdam, The Netherlands, ${ }^{4}$ Department of Thrombosis Research, Ribe County Hospital, University of Southern Denmark and Department of Clinical \\ Biochemistry, 6700 Esbjerg, Denmark, ${ }^{5}$ Department of Clinical Biochemistry, Frederiksberg Hospital, 2000 Frederiksberg, Denmark, ${ }^{6}$ Faculty of Health \\ Sciences, University of Aarhus, 8000 Aarhus, Denmark, ${ }^{7}$ Department of Medical Endocrinology, University of Copenhagen, Rigshospitalet, 2000 \\ Copenhagen, Denmark and ${ }^{8}$ Department of Endocrinology, University of Lund, S-20502 Malmö, Sweden
}

(Correspondence should be addressed to S S Lund; Email: sqrl@steno.dk)

\begin{abstract}
Objective: In patients with type 2 diabetes mellitus (T2DM), biomarkers reflecting inflammation and endothelial dysfunction have been linked to cardiovascular disease (CVD biomarkers) and metabolic regulation. In T2DM patients, metformin and insulin secretagogues have demonstrated equal antihyperglycaemic potency. Here, we report the effect of metformin versus an insulin secretagogue, repaglinide, on CVD biomarkers in non-obese T2DM patients.

Design and methods: Single-centre, double-masked, double-dummy, crossover study during $2 \times 4$ months involving 96 non-obese (body mass index $\leq 27 \mathrm{~kg} / \mathrm{m}^{2}$ ) insulin-naïve T2DM patients. At enrolment, previous oral hypoglycaemic agents were stopped and the patients entered a 1-month runin on diet-only treatment. Hereafter, patients were randomized to either $2 \mathrm{mg}$ repaglinide thrice daily followed by $1 \mathrm{~g}$ metformin twice daily or vice versa each during 4 months with a 1-month washout between interventions.

Results: Levels of tumour necrosis factor- $\alpha$, plasminogen activator inhibitor-1 antigen, tissue-type plasminogen activator antigen, von Willebrand factor, soluble intercellular adhesion molecule- 1 and soluble E-selectin were significantly lower during metformin versus repaglinide treatments. In contrast, Amadori albumin and heart rate were higher during metformin versus repaglinide. Levels of interleukin-6, fibrinogen, soluble vascular cell adhesion molecule-1, asymmetric dimethylarginine and advanced glycation end products as well as glycaemic levels (previously reported) and 24-h blood pressure were similar between treatments. Adjustment for known macrovascular disease did not affect the between-treatment effects.

Conclusions: In non-obese T2DM patients, metformin was more effective in reducing selected biomarkers reflecting inflammation and endothelial dysfunction compared with repaglinide despite similar glycaemic levels between treatments.
\end{abstract}

European Journal of Endocrinology 158 631-641

\section{Background}

Patients with type 2 diabetes mellitus (T2DM) have an increased risk of cardiovascular disease (CVD) when compared with individuals without diabetes (1). Conventional risk factors such as dyslipidaemia, smoking and/or hypertension do not fully explain the extent of cardiovascular mortality in patients with diabetes (2). Mechanisms related to inflammation as well as endothelial function have been implicated in the pathophysiological process leading from the formation of atherosclerotic plaques to clinical events of thrombosis in patients with diabetes (3). In accordance with these concepts, markers reflecting inflammation (e.g. C-reactive protein (CRP), interleukin-6 (IL-6), tumour necrosis factor- $\alpha$ (TNF- $\alpha$ ) and fibrinogen) and endothelial dysfunction (e.g. von Willebrand factor (vWf), soluble adhesion molecules, asymmetric dimethylarginine (ADMA), tissue-type plasminogen activator, plasminogen activator inhibitor- 1 and albuminuria) have been shown, independent of conventional risk factors, to be associated with the risk of CVD and/or CVD mortality in individuals with T2DM, nondiabetic subjects and/or the general population (4-13). 
Also, biomarkers related to adiposity and insulin sensitivity (e.g. adiponectin) as well as glycation of proteins (e.g. Amadori products and advanced glycation end products (AGE)) have been linked with the atherosclerotic processes $(11,14,15)$. Here, we collectively term these non-conventional CVD risk markers 'CVD biomarkers'. Interrelations between the CVD biomarkers and the glycaemic variables have been suggested (16-18). Thus, biological mechanisms both dependent and independent of glycaemia might influence the CVD biomarkers. As yet, few studies have compared the effect of various anti-hyperglycaemic treatment regimens on CVD biomarkers, and most studies were in obese patients with T2DM $(17,19-27)$.

Metformin is an oral anti-hyperglycaemic agent which enhances insulin sensitivity and lowers hepatic glucose output (28). In obese patients with T2DM, metformin is currently the drug of first choice due to its bilateral effect on glycaemic regulation and cardiovascular protection $(29,30)$. However, obese and non-obese patients with T2DM experience a similar cardiovascular risk $(31,32)$ and the use of metformin even in the non-obese patients with T2DM might be beneficial as well. Recently, in non-obese patients with T2DM, we demonstrated equal anti-hyperglycaemic potency between metformin and the insulin secretagogue repaglinide (33). Repaglinide is a glibenclamide moiety belonging to the short-acting meglitinide analogue insulin secretagogues. Repaglinide has demonstrated similar anti-hyperglycaemic potency compared with sulphonylurea insulin secretagogues (34). In addition, repaglinide has shown improved potency on clinical CVD surrogate markers when compared with sulphonylurea insulin secretagogues (e.g. a decrease in carotid intima-media thickness and inflammatory markers with repaglinide versus glyburide, i.e. glibenclamide, (23) as well as improved brachial artery reactivity with repaglinide versus glibenclamide (35)). Whether metformin has equal potency on potentially important CVD biomarkers when compared with an insulin secretagogue in non-obese patients with T2DM is unknown. We therefore aimed to investigate the effect of metformin versus repaglinide treatment on CVD biomarkers in insulin-naïve non-obese patients with T2DM.

\section{Materials and methods}

The study design as well as the results of glycaemic regulation have been published in details previously (33). Briefly, it was an investigator-initiated, singlecentre, randomized, double-masked, double-dummy, crossover study of 96 non-obese (body mass index $(\mathrm{BMI}) \leq 27 \mathrm{~kg} / \mathrm{m}^{2}$ ) insulin-naïve patients with T2DM. The diagnosis of T2DM was established if patients were older than 40 years of age at the onset of diabetes, had no history of ketonuria or diabetic ketoacidosis and, at enrolment, presented with a fasting serum C-peptide $\geq 300 \mathrm{pmol} / \mathrm{l}$ or a non-fasting or glucagon-stimulated C-peptide $\geq 600 \mathrm{pmol} / \mathrm{l}$. At enrolment, patients stopped previous oral hypoglycaemic agents (OHA) and began a 1-month run-in period on diet-only treatment. Patients with haemoglobin $\mathrm{A}_{1 \mathrm{c}}\left(\mathrm{HbA}_{1 \mathrm{c}}\right) \geq 6.5 \%$ after the run-in period were randomized to treatment sequences of either $2 \mathrm{mg}$ repaglinide thrice daily followed by $1 \mathrm{~g}$ metformin twice daily, or vice versa, each for a period of 4 months with a 1-month washout between interventions. The primary end point was $\mathrm{HbA}_{1 \mathrm{c}}$ and the secondary end points were other glycaemic variables and CVD biomarkers. Blood pressure, albuminuria, as well as markers of inflammation and endothelial dysfunction were measured during the last week before the patient entered a treatment period and on the last day of each treatment period. Of the 96 randomized patients, $20(21 \%)$ dropped out, leaving 76 patients $(79 \%)$ who completed the trial $(83$ and 82 patients completed a treatment period with metformin and repaglinide respectively). Ten (11\%) versus seven $(8 \%)$ patients were excluded during metformin and repaglinide treatments respectively. Three (3\%) further patients dropped out during the washout period.

The study was conducted in accordance with the Declaration of Helsinki and was approved by the Ethics Committee of Copenhagen County, Denmark.

Data on $\mathrm{HbA}_{1 \mathrm{c}}$, additional glycaemic variables, highsensitivity CRP (hsCRP) and adiponectin have been reported elsewhere (33).

\section{Blood sampling}

Patients were investigated at the Steno Diabetes Center, Gentofte, Denmark. Blood samples were drawn with minimal venous occlusion between 0800 and $0900 \mathrm{~h}$ after a 10-h overnight fast and 25-min supine rest. Plasma and serum samples were prepared by centrifugation at $2000 \boldsymbol{g}$ for $10 \mathrm{~min}$ at $20^{\circ} \mathrm{C}$ and immediately frozen hereafter. The samples for measurement of circulating biomarkers were stored at $-80{ }^{\circ} \mathrm{C}$ until analysis.

\section{Biochemical analyses and other}

Commercially available ELISA kits were used for the measurements of serum levels of soluble vascular cell adhesion molecule-1 (sVCAM-1), soluble intercellular adhesion molecule-1 (sICAM-1), soluble E-selectin (sE-selectin) (Diaclone, Besançon, France), IL-6 (Quantikine High Sensitivity; R\&D Systems, Oxon, UK) and adiponectin (ACRP30, Linco Research, St Charles, MO, USA). Plasma samples for measurement of fibrinogen (MultifibrenU; Dade Behring, Marburg, Germany), tissue-type plasminogen activator antigen (t-PA-ag) (Immulyse t-PA; Biopool, Umeå, Sweden), Amadori albumin (ELISA (36)) and vWf antigen were collected in $4.5 \mathrm{ml}$ vacutainer tubes containing natrium citrate $129 \mathrm{mM}$. Levels of vWf antigen and serum CRP were 
determined by highly sensitive enzyme immunoassays (37). Levels of $\mathrm{vWf}$ are expressed as percentage of antigen levels in normal pooled plasma. The plasma samples for measurement of plasminogen activator inhibitor-1 antigen (PAI-1-ag) (TintElize PAI-1; Biopool) were collected in $5 \mathrm{ml}$ vacutainer containing a premixed solution of $0.5 \mathrm{ml}$ of $110 \mathrm{mM}$ citrate monohydrate, $15 \mathrm{mM}$ teophyllin, $3.7 \mathrm{mM}$ adenosine and $0.198 \mathrm{mM}$ dipyridamol. ADMA (HPLC (38)), TNF- $\alpha$ (Quantikine High Sensitivity; R\&D Systems), AGE peptides, $\mathrm{N}^{\varepsilon}$-carboxymethyllysine (CML) and $\mathrm{N}^{\varepsilon}$-carboxyethyl lysine (CEL) were measured in EDTA plasma. AGE peptides were measured according to the method described by Wrobel et al. (39). Levels of CML and CEL were measured by stable-isotope dilution tandem mass spectrometry (40). Urinary albumin excretion rate (UAER) was estimated from a single 24-h urine sample by turbidimetry (Hitachi 912, Roche Diagnostics, Mannheim, Germany). Twenty-four-hour ambulatory blood pressure and heart rate measurements were performed with the Takeda TM-2421 device (A\&D Instruments, Tokyo, Japan) performing automated measurements with 15-min intervals during the daytime (from 0700 to $2259 \mathrm{~h}$ ) and 30-min intervals during the night-time (from 2300 to $0659 \mathrm{~h}$ ). The measurements were averaged over each hour before statistical analysis. Mean arterial blood pressure was defined as diastolic blood pressure plus one-third of the pulse pressure (i.e. the difference between systolic and diastolic blood pressures). At enrolment, office blood pressure was measured with a digital automatic device UA-779 (A\&D Instruments Ltd, Abingdon, Oxon, UK), one measurement on each arm after a minimum of 5 -min rest in the upright sitting position. The mean of the two measurements was used as the level of office blood pressure at enrolment. Macrovascular disease was defined as known atherosclerotic disease (e.g. stroke, ischaemic heart disease or peripheral arterial disease). Microalbuminuria and macroalbuminuria were defined as an UAER 30-299 or $\geq 300 \mathrm{mg} / \mathrm{d}$ respectively, in two out of three consecutive 24-h urine collections prior to enrolment. Neuropathy was defined as symptomatic or clinical signs of peripheral or autonomic neuropathy. Data on retinopathy was obtained from patient records.

\section{Statistical analysis}

We evaluated the randomized population $(n=96$ patients). However, for treatment effects, only patients who completed at least one treatment period $(n=89$ patients) were included in the analysis. Each outcome was evaluated after the run-in period (before initiating treatment in the first treatment period), referred to as the 'first-period baseline', and at the end of each treatment period, referred to as 'end of treatment'. The target parameter was differences in treatment effects between interventions, evaluated by comparisons of end of treatment levels from both treatment periods with that of the first-period baseline (i.e. "change from first-period baseline').

Data were analysed with a linear normal mixed model with the subject as a random effect. Treatment type (metformin or repaglinide), treatment sequence (metformin followed by repaglinide or vice versa), the period effect and the first-period baseline were included as fixed effects. This model enables information from incomplete blocks, i.e. those with dropouts, to be included when estimating treatment effects (both within- and betweensubject information were included) (41). In a two-period crossover study, it is not possible to estimate carry-over phenomena (42). We had no prior suspicion of carryover phenomena at the end of the trial (i.e. 5 months after cessation of the first-period treatment). We have therefore assumed a no carry-over situation, which has been considered as a reliable approach in a two-period crossover study (43). However, by using the model as outlined above, the potential occurences of any unexpected carry-over phenomena at the end of the trial have been adjusted for to the degree such phenomena influenced the effects of period and/or treatment sequence. Also, the degree to which the change from first-period baseline was influenced by the effect of period has likewise been adjusted for by this model. Thus, all given within- (i.e. change from firstperiod baseline) and between-treatment estimates are readily comparable. The effect of subgroups of patients according to previously diagnosed macrovascular disease was examined by interaction analyses as well as adjustment for subgroup. The effect of metabolic variables (i.e. $\mathrm{HbA}_{1 \mathrm{c}}$, body weight and fasting serum insulin) was examined by adjustment for their current levels (i.e. first-period baseline and on-treatment).

Data are presented as raw values (i.e. without statistical modelling) either given as mean (s.D.) or, for non-normally distributed variables, as the median or geometric mean (range). Treatment effects are reported as derived by the model given as means ( $95 \%$ confidence intervals (CI)). End points with non-normally distributed residuals or random effects were logarithmic transformed before analysis. The treatment effects for these end points are reported as percentage changes. For AGE peptides, untransformed or logarithmic transformed data did not show normally distributed residuals. AGE peptides data were therefore analyzed with a non-parametric Wilcoxon signed ranks test. The level of significance was 5\% (two sided).

All statistical analyses were done with SPSS v. 14.0 (Chicago, IL, USA).

\section{Results}

Details of patient characteristics at enrolment have been presented elsewhere (33). Briefly, the 96 randomized patients were all Caucasians and predominantly men (women: $n=23$; men: $n=73$ ). At enrolment, their 
mean age was 61.4 (9.3) years and median known duration of T2DM was $4(0 ; 28)$ years. Mean BMI was $24.8(2.0) \mathrm{kg} / \mathrm{m}^{2}$ and mean $\mathrm{HbA}_{1 \mathrm{c}}$ was $7.45 \%(0.85)$ at pre-study anti-hyperglycaemic treatment (diet-only, $n=16$; OHA monotherapy, $n=65$; OHA combination therapy: $n=15)$. Mean office blood pressure was $146.5 \mathrm{mmHg}$ (17.8) systolic and $82.2 \mathrm{mmHg}$ (9.6) diastolic. A total of $30(31 \%)$ patients presented with retinopathy and 27 (28\%) patients with micro- or macro-albuminuria. A total of 21 (22\%) patients had known macrovascular disease and $70(73 \%)$ patients presented with neuropathy. At enrolment, $52(54 \%)$ patients received anti-hypertensive therapy, 43 (45\%) patients received aspirin and $29(30 \%)$ patients received lipid-lowering treatment as concomitant non-study medication (33). First-period baseline data are summarized in Tables 1 and 2. A total of five patients (5\%) had positive glutamic acid decarboxylase-65 (GAD65) antibody titres (above $31 \mathrm{U} / \mathrm{ml}$ ). Also, a total of five patients $(5 \%)$ had fasting serum C-peptide levels below $300 \mathrm{pmol} / \mathrm{l}$ at first-period baseline. Hereof, four patients demonstrated non-fasting serum C-peptide levels above $600 \mathrm{pmol} / \mathrm{l}$ and, upon re-test, one patient demonstrated fasting serum C-peptide levels above $300 \mathrm{pmol} / \mathrm{l}$. Also, among these five patients, four of them were GAD65 antibody negative and one patient (a dropout patient) had missing value (33).
After close-out, genotyping was performed. One patient was diagnosed with maturity onset diabetes of the young type 3 (MODY-3). The patient presented with a fasting C-peptide of $574 \mathrm{pmol} / \mathrm{l}$ at first-period baseline. The patients were not screened for maternally inherited diabetes and deafness, but no patient presented with signs of hearing loss).

\section{Endpoints}

During metformin treatment levels of TNF- $\alpha$, PA1-ag, t-PA-ag, vWf, sICAM-1 and sE-selectin were significantly lower when compared with repaglinide. In contrast, Amadori albumin and heart rate were higher with metformin versus repaglinide. The change in IL-6, fibrinogen, sVCAM-1, ADMA as well as 24-h blood pressure and albuminuria were not significantly different between treatments (Table 3). Levels of AGE peptides, CML and CEL did not change significantly during either treatment compared with first-period baseline or between treatments (data not shown).

The test for interaction (heterogeneity) of study-drug treatment by the presence $(n=21) /$ absence $(n=75)$ of known macrovascular disease was not significant for any of the investigated variables. Also, adjustment for the presence/absence of known macrovascular disease did not change the overall between-treatment

Table 1 First-period baseline data for variables associated with cardiovascular disease risk in non-obese patients with type 2 diabetes (data are obtained after a 1-month washout with diet-only treatment after stopping pre-study anti-hyperglycaemic treatment).

\begin{tabular}{|c|c|}
\hline Endpoint & $\begin{array}{l}\text { First-period baseline } \\
\text { Mean (S.D.) or geometric mean (range) }(n=96)\end{array}$ \\
\hline \multicolumn{2}{|l|}{ Biomarkers of inflammation } \\
\hline Serum high sensitive C-reactive protein (mg/l) & $2.07(0.12 ; 26.47)$ \\
\hline Plasma tumour necrosis factor- $\alpha(\mathrm{pg} / \mathrm{ml})(n=94)^{\mathrm{a}}$ & $3.24(1.62 ; 30.30)$ \\
\hline Serum interleukin-6 (pg/ml) & $2.47(0.69 ; 288.75)$ \\
\hline Plasma fibrinogen $(\mu \mathrm{mol} / \mathrm{l})$ & $10.0(6.8 ; 15.5)$ \\
\hline \multicolumn{2}{|l|}{ Biomarkers of endothelial dysfunction } \\
\hline Plasma plasminogen activator inhibitor-1 antigen (ng/ml) & $17.3(3.0 ; 114.1)$ \\
\hline Plasma tissue-type plasminogen activator antigen $(\mathrm{ng} / \mathrm{ml})$ & $12.43(5.28)$ \\
\hline Plasma von Willebrand factor (\%) & $114(38 ; 280)$ \\
\hline Serum soluble intercellular adhesion molecules-1 (ng/ml) & $518(199 ; 1256)$ \\
\hline Serum soluble vascular cell adhesion molecules-1 (ng/ml) & $833(440 ; 2177)$ \\
\hline Serum soluble E-selectin $(\mathrm{ng} / \mathrm{ml})$ & $83(19 ; 284)$ \\
\hline \multicolumn{2}{|l|}{ Other biomarkers } \\
\hline Plasma asymmetric dimethylarginine $(\mu \mathrm{mol} / \mathrm{l})$ & $0.473(0.058)$ \\
\hline Plasma Amadori albumin ( $\mu \mathrm{mol} / \mathrm{l})$ & $50.33(11.52)$ \\
\hline Plasma advanced glycation end products (\%) & $12.8(8.0 ; 20.3)$ \\
\hline Plasma $N^{\varepsilon}$-carboxymethyllysine ( $\mu \mathrm{mol} / /$ per $\mathrm{mmol} / / \mathrm{l}$ Lysine $)$ & $0.069(0.015)$ \\
\hline Plasma $N^{\varepsilon}$-carboxyethyllysine ( $\mu \mathrm{mol} / / \mathrm{l}$ per mmol// Lysine) & $0.016(0.003)$ \\
\hline Serum adiponectin $(\mu \mathrm{g} / \mathrm{ml})$ & $5.8(2.5 ; 19.5)$ \\
\hline \multicolumn{2}{|l|}{ 24-h ambulatory blood pressure and heart-rate } \\
\hline Systolic blood pressure $(\mathrm{mmHg})$ & $132.4(13.7)$ \\
\hline Diastolic blood pressure $(\mathrm{mmHg})$ & $72.1(7.5)$ \\
\hline Mean arterial blood pressure $(\mathrm{mmHg})$ & $92.2(8.5)$ \\
\hline Heart rate (beats/min) & $74.0(8.6)$ \\
\hline \multicolumn{2}{|l|}{ Albuminuria } \\
\hline Urinary albumin excretion rate $(\mathrm{mg} / 24 \mathrm{~h})(n=95)^{\mathrm{a}}$ & $17(3 ; 1123)$ \\
\hline
\end{tabular}


Table 2 First-period baseline data for variables associated with cardiovascular disease risk in a total of 89 non-obese patients with type 2 diabetes (data are obtained after a 1-month washout with diet-only treatment after stopping pre-study anti-hyperglycaemic treatment).

First-period baseline

Mean (S.D.) or geometric mean (range)

\section{Endpoint}

Metformin $(n=83)$

Repaglinide $(n=82)$

Biomarkers of inflammation

Serum high sensitive C-reactive protein $(\mathrm{mg} / \mathrm{l})$

Plasma tumour necrosis factor- $\alpha(\mathrm{pg} / \mathrm{ml})(n=88)^{\mathrm{a}}$

Serum interleukin-6 $(\mathrm{pg} / \mathrm{ml})$

Plasma fibrinogen $(\mu \mathrm{mol} / \mathrm{l})$

Biomarkers of endothelial dysfunction

Plasma plasminogen activator inhibitor-1 antigen $(\mathrm{ng} / \mathrm{ml})$

Plasma tissue-type plasminogen activator antigen $(\mathrm{ng} / \mathrm{ml})$

Plasma von Willebrand factor (\%)

Serum soluble intercellular adhesion molecules-1 $(\mathrm{ng} / \mathrm{ml})$

Serum soluble vascular cell adhesion molecules-1 $(\mathrm{ng} / \mathrm{ml})$

Serum soluble E-selectin $(\mathrm{ng} / \mathrm{ml})$

Other biomarkers

Plasma asymmetric dimethylarginine $(\mu \mathrm{mol} / \mathrm{l})$

Plasma Amadori albumin ( $\mu \mathrm{mol} / \mathrm{l})$

Plasma advanced glycation end products (\%)

Plasma $N^{\varepsilon}$-carboxymethyllysine ( $\left.\mu \mathrm{mol} / / \mathrm{per} \mathrm{mmol} / / \mathrm{Lysine}\right)$

Plasma $N^{\varepsilon}$-carboxyethyllysine ( $\mu \mathrm{mol} / /$ per $\left.\mathrm{mmol} / / \mathrm{Lysine}\right)$

Serum adiponectin $(\mu \mathrm{g} / \mathrm{ml})$

24-h ambulatory blood pressure and heart-rate

Systolic blood pressure $(\mathrm{mmHg})$

Diastolic blood pressure $(\mathrm{mmHg})$

Mean arterial blood pressure $(\mathrm{mmHg})$

Heart rate (beats/min)

Albuminuria

Urinary albumin excretion rate $(\mathrm{mg} / 24 \mathrm{~h})(n=88)^{\mathrm{a}}$

$$
\begin{aligned}
& 1.95(0.12 ; 26.47) \\
& 3.23(1.62 ; 30.30) \\
& 2.39(0.69 ; 288.75) \\
& 9.9(6.8 ; 15.5)
\end{aligned}
$$

$$
\begin{aligned}
18.4 & (3.0 ; 114.1) \\
12.04 & (4.97) \\
114 & (38 ; 280) \\
516 & (199 ; 1256) \\
822 & (440 ; 2177) \\
82 & (19 ; 284)
\end{aligned}
$$

$0.472(0.059)$

$49.16(11.50)$

$12.7(8.0 ; 20.3)$

$0.069(0.015)$

$0.016(0.003)$

$5.6(2.5 ; 19.5)$

$131.4(13.5)$

$71.9(7.4)$

$91.8(8.4)$

$74.7(8.7)$

$16(3 ; 1123)$
$2.00(0.12 ; 26.47)$

$3.19(1.62 ; 30.30)$

$2.38(0.69 ; 288.75)$

$9.8(6.8 ; 15.4)$

17.1 (3.0; 114.1)

$11.97(5.28)$

$113(38 ; 280)$

$511(199 ; 1256)$

$816(440 ; 2177)$

$81(19 ; 284)$

$0.474(0.058)^{\mathrm{b}}$

$49.10(11.27)$

$12.9(8.0 ; 20.3)$

$0.069(0.015)$

$0.016(0.003)$

$5.6(2.5 ; 19.5)$

$132.0(13.7)$

$71.9(7.8)$

$91.9(8.6)$

73.7 (8.5)

15 (3; 268)

Data for metformin and repaglinide represent patients who completed a treatment period with either drug (metformin, $n=83$; repaglinide, $n=82$; total, $n=89$ ). Hence, identical first-period baseline data from patients who completed a treatment period with both drugs $(n=76)$ are included in the metformin as well as in the repaglinide groups (i.e. such patients are included twice in the table). Moreover, except explicit stated (see note b, below), first-period baseline data are also included for patients who completed one or both treatment periods, but despite this had missing values at end of treatment (i.e. missing values at end of treatment could appear due to, e.g. technical difficulties with analytical assays or other). For first-period baseline data in all 96 randomized patients with each patient included only once, please see Table 1. All plasma and serum variables were measured in the fasting state. For non-normally distributed variables, geometric mean (range) values are given with the number of measured decimals, whereas for normally distributed variables measured with less than two decimals, mean (s.D.) values are given with one additional decimal.

${ }^{a}$ Number of patients included if different from $n=89$.

${ }^{\mathrm{b}}$ The mean (S.D.) first-period baseline levels for asymmetric dimethylarginine according to the number of patients with available data at end of treatment was metformin $(n=82): 0.472 \mu \mathrm{mol} / \mathrm{l}(0.059)$ and repaglinide $(n=80): 0.472 \mu \mathrm{mol} / \mathrm{l}(0.057)$.

effects substantially for any of the investigated variables (data not shown).

When adjusted for the current levels of $\mathrm{HbA}_{1 \mathrm{c}}$, body weight and fasting serum insulin, the levels of TNF- $\alpha$, t-PA-ag, sE-selectin and Amadori albumin were no longer significantly different between treatments, whereas levels of sVCAM-1 became significantly lower during metformin versus repaglinide (mean $(95 \%$ CI) percentage difference in the levels of sVCAM-1 during metformin versus repaglinide adjusted for the current levels of $\mathrm{HbA}_{1 \mathrm{c}}$, body weight and fasting serum insulin: $-3 \%(-5 ;-0), P=0.033)$. The between-treatment effect for other variables did not change substantially. Also, levels of sVCAM-1 were significantly lower during metformin versus repaglinide after exclusion of the five patients diagnosed with positive GAD65 antibody titres (mean $(95 \%$ CI) percentage difference in the levels of sVCAM-1 during metformin versus repaglinide after exclusion of five patients with positive GAD65 antibody titres: $-3 \%(-5 ;-0), P=0.029)$. Otherwise, other variables did not change substantially after exclusion of either the five patients diagnosed with positive GAD65 antibody titres or the patient diagnosed with MODY-3 (data not shown).

\section{Discussion}

In 96 non-obese patients with T2DM treated for 4 months with either metformin or repaglinide, we found significantly lower levels of several circulating non-glycaemic biomarkers associated with the risk of CVD (TNF- $\alpha$, PAI-1-ag, t-PA-ag, vWf, sICAM-1 and sE-selectin) (4, 6-9) during metformin compared with repaglinide treatments. In contrast, heart rate and Amadori albumin were significantly higher during treatment with metformin versus treatment with repaglinide with no difference in 24-h blood pressure, albuminuria, AGE peptides, CML or CEL between treatments. The observed effects were independent of whether individuals had known macrovascular disease. 
Table 3 Comparison of effects of metformin and repaglinide on variables associated with cardiovascular disease risk in non-obese patients with type 2 diabetes.

\begin{tabular}{|c|c|c|c|c|c|c|}
\hline \multirow[b]{2}{*}{ Endpoint } & \multicolumn{2}{|c|}{$\begin{array}{l}\text { End of treatment }{ }^{\mathrm{a}} \\
\text { Mean (s.D.) or geometric mean (range) }\end{array}$} & \multicolumn{2}{|c|}{$\begin{array}{l}\text { Change from first-period baseline }{ }^{a} \\
\text { Mean ( } 95 \% \text { confidence interval) }\end{array}$} & \multicolumn{2}{|c|}{$\begin{array}{l}\text { Between treatment effect }{ }^{\mathrm{a}} \\
\text { Mean ( } 95 \% \text { confidence interval) }\end{array}$} \\
\hline & $\begin{array}{l}\text { Metformin } \\
(n=83)^{\mathrm{b}}\end{array}$ & $\begin{array}{l}\text { Repaglinide } \\
\qquad(n=82)^{\mathrm{b}}\end{array}$ & $\begin{array}{l}\Delta \text { Metformin } \\
(n=83)^{\mathrm{b}}\end{array}$ & $\begin{array}{l}\Delta \text { Repaglinide } \\
\quad(n=82)^{\mathrm{b}}\end{array}$ & $\begin{array}{l}\text { Metformin versus } \\
\text { Repaglinide }(n=89)^{\mathrm{b}}\end{array}$ & $P$ \\
\hline \multicolumn{7}{|l|}{ Biomarkers of inflammation } \\
\hline $\begin{array}{l}\text { Serum high sensitive C-reactive protein } \\
(\mathrm{mg} /)^{\mathrm{c}}(n=88)^{\mathrm{d}}\end{array}$ & $1.50(0.07 ; 26.90)$ & $1.80(0.07 ; 40.77)$ & $-23(-37 ;-6)^{\mathrm{e}}$ & $-8(-24 ; 13)^{\mathrm{e}}$ & $-17(-34 ; 5)^{\dagger}$ & 0.125 \\
\hline $\begin{array}{l}\text { Plasma tumour necrosis factor- } \alpha(\mathrm{pg} / \mathrm{ml})^{\mathrm{c}} \\
(n=86)^{\mathrm{d}}\end{array}$ & $3.04(1.35 ; 9.15)$ & $3.21(1.59 ; 17.20)$ & $-4(-9 ; 2)^{\mathrm{e}}$ & $4(-2 ; 11)^{\mathrm{e}}$ & $-8(-14 ;-1)^{f}$ & 0.027 \\
\hline $\begin{array}{l}\text { Serum interleukin- } 6(\mathrm{pg} / \mathrm{ml})^{\mathrm{c}}(n=88)^{\mathrm{d}} \\
\text { Plasma fibrinogen }(\mu \mathrm{mol} / \mathrm{l})(n=88)^{\mathrm{d}}\end{array}$ & $\begin{array}{l}2.28(0.61 ; 212.88) \\
9.6(6.9 ; 15.0)\end{array}$ & $\begin{array}{l}2.49(0.71 ; 164.00) \\
9.6(6.8 ; 16.0)\end{array}$ & $\begin{array}{l}-4(-13 ; 6)^{\mathrm{e}} \\
-4(-7 ;-0)^{\mathrm{e}}\end{array}$ & $\begin{array}{r}6(-5 ; 17)^{\mathrm{e}} \\
-2(-6 ; 1)^{\mathrm{e}}\end{array}$ & $\begin{array}{l}-9(-20 ; 3)^{f} \\
-1(-5 ; 3)^{f}\end{array}$ & $\begin{array}{l}0.124 \\
0.519\end{array}$ \\
\hline \multicolumn{7}{|l|}{ Biomarkers of endothelial dysfunction } \\
\hline $\begin{array}{l}\text { Plasma plasminogen activator } \\
\text { inhibitor-1 antigen }(\mathrm{ng} / \mathrm{ml})^{\mathrm{c}}(n=88)^{\mathrm{d}}\end{array}$ & $17.4(<0.9 ; 78.4)$ & $22.7(<0.9 ; 99.0)$ & $-3(-16 ; 12)^{\mathrm{e}}$ & $30(12 ; 51)^{\mathrm{e}}$ & $-25(-37 ;-12)^{f}$ & 0.001 \\
\hline $\begin{array}{l}\text { Plasma tissue-type plasminogen activator } \\
\text { antigen }(\mathrm{ng} / \mathrm{ml})(n=88)^{\mathrm{d}}\end{array}$ & $10.23(4.66)$ & $11.61(5.03)$ & $-1.76(-2.67 ;-0.84)$ & $-0.33(-1.26 ; 0.60)$ & $-1.43(-2.64 ;-0.22)$ & 0.021 \\
\hline Plasma von Willebrand factor $(\%)(n=88)^{\mathrm{d}}$ & $102(45 ; 258)$ & $109(43 ; 262)$ & $-11(-14 ;-8)^{\mathrm{e}}$ & $-3(-7 ; 0)^{\mathrm{e}}$ & $-8(-11 ;-4)^{\dagger}$ & $<0.001$ \\
\hline $\begin{array}{l}\text { Serum soluble intercellular adhesion } \\
\text { molecules- } 1(\mathrm{ng} / \mathrm{ml})^{\mathrm{C}}(n=88)^{\mathrm{d}}\end{array}$ & $479(197 ; 1278)$ & $501(221 ; 1718)$ & $-7(-10 ;-4)^{e}$ & $-2(-4 ; 1)^{\mathrm{e}}$ & $-6(-8 ;-3)^{f^{\prime}}$ & $<0.001$ \\
\hline $\begin{array}{l}\text { Serum soluble vascular cell adhesion } \\
\text { molecules- } 1(\mathrm{ng} / \mathrm{ml})^{\mathrm{c}}(n=88)^{\mathrm{d}}\end{array}$ & $773(430 ; 2574)$ & $783(398 ; 2584)$ & $-6(-8 ;-4)^{e}$ & $-4(-6 ;-2)^{\mathrm{e}}$ & $-2(-5 ; 0)^{f}$ & 0.068 \\
\hline Serum soluble E-selectin $(\mathrm{ng} / \mathrm{ml})^{\mathrm{c}}(n=88)^{\mathrm{d}}$ & $73(22 ; 230)$ & $75(23 ; 283)$ & $-11(-15 ;-7)^{\mathrm{e}}$ & $-7(-11 ;-3)^{\mathrm{e}}$ & $-4(-8 ;-0)^{f}$ & 0.033 \\
\hline \multicolumn{7}{|l|}{ Other biomarkers } \\
\hline $\begin{array}{l}\text { Plasma asymmetric dimethylarginine } \\
(\mu \mathrm{mol} / \mathrm{l})(n=88)^{\mathrm{d}}\end{array}$ & $0.475(0.055)$ & $0.473(0.055)$ & $0.003(-0.005 ; 0.011)$ & $0.000(-0.008 ; 0.007)$ & $0.003(-0.006 ; 0.013)$ & 0.497 \\
\hline Plasma Amadori albumin $(\mu \mathrm{mol} / \mathrm{l})(n=88)^{\mathrm{d}, \mathrm{g}}$ & $45.62(8.44)$ & $43.56(11.46)$ & $-3.39(-4.85 ;-1.93)$ & $-5.48(-6.96 ;-4.00)$ & $2.09(0.43 ; 3.76)$ & 0.015 \\
\hline Serum adiponectin $(\mu \mathrm{g} / \mathrm{ml})^{\mathrm{c}}(n=88)^{\mathrm{d}}$ & $5.9(2.9 ; 23.4)$ & $5.9(3.0 ; 29.3)$ & $5(1.9)^{\mathrm{e}}$ & $5(0 ; 9)^{\mathrm{e}}$ & $0(-4 ; 5)^{f}$ & 0.882 \\
\hline \multicolumn{7}{|c|}{ 24-h ambulatory blood pressure and heart rate } \\
\hline Systolic blood pressure $(\mathrm{mmHg})(n=88)^{\mathrm{d}, \mathrm{g}}$ & $132.4(15.9)$ & $132.3(14.9)$ & $1.0(-1.6 ; 3.7)$ & $0.5(-2.2 ; 3.2)$ & $0.5(-2.3 ; 3.4)$ & 0.715 \\
\hline Diastolic blood pressure $(\mathrm{mmHg})(n=88)^{\mathrm{d}, \mathrm{g}}$ & $71.2(7.4)$ & $71.4(7.5)$ & $-0.8(-2.1 ; 0.5)$ & $-0.5(-1.8 ; 0.8)$ & $-0.3(-1.5 ; 0.9)$ & 0.626 \\
\hline $\begin{array}{l}\text { Mean arterial blood pressure }(\mathrm{mmHg}) \\
(n=88)^{\mathrm{d}, \mathrm{g}}\end{array}$ & $91.6(9.1)$ & $91.7(8.7)$ & $-0.2(-1.7 ; 1.3)$ & $-0.2(-1.7 ; 1.4)$ & $-0.0(-1.5 ; 1.5)$ & 0.982 \\
\hline Heart rate $($ beats $/ \min )(n=88)^{\mathrm{d}, \mathrm{g}}$ & $75.7(8.9)$ & $73.6(8.4)$ & $1.2(0.1 ; 2.4)$ & $-0.2(-1.3 ; 1.0)$ & $1.4(0.1 ; 2.7)$ & 0.040 \\
\hline \multicolumn{7}{|l|}{ Albuminuria } \\
\hline $\begin{array}{l}\text { Urinary albumin excretion rate }(\mathrm{mg} / 24 \mathrm{~h})^{\mathrm{c}} \\
(n=87)^{\mathrm{d}}\end{array}$ & $17(2 ; 1737)$ & $14(2 ; 1186)$ & $4(-10 ; 21)^{\mathrm{e}}$ & $-5(-18 ; 10)^{\mathrm{e}}$ & $10(-4 ; 27)^{f}$ & 0.159 \\
\hline
\end{tabular}

All plasma and serum variables were measured in the fasting state. Data are not shown for advanced glycation end products, $\mathrm{N}^{\varepsilon}$-carboxymethyllysine and $\mathrm{N}^{\varepsilon}$-carboxyethyllysine. a End of treatment levels represent raw absolute values, whereas changes from first-period baseline and between-treatment effects represent estimates derived from the model. bNumbers in brackets refer to number of patients included in the statistical model unless otherwise is stated in the endpoint name column.

${ }^{c}$ Data are natural logarithmic transformed prior to the analysis of changes from first-period baseline and between-treatment effects. Percentage differences are shown with no decimals.

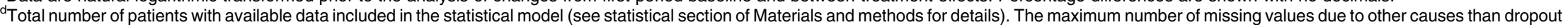
(e.g. due to technical difficulties with the analytical assays or other) was observed for tumour necrosis factor- $\alpha$ (TNF- $\alpha$ ) for metformin as well as for repaglinide (number of patients with available data on TNF- $\alpha$ at end of treatment: metformin: $n=81$ and repaglinide: $n=78$ ). Otherwise, the numbers of patients in each of the two treatment groups at end of treatment and as changes from first-period baseline effects are not shown.

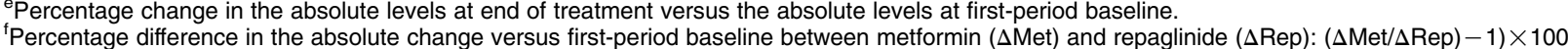

${ }^{9}$ For variables measured with less than two decimals, mean (s.D.) and mean $(95 \% \mathrm{Cl})$ values are given with one additional decimal. 
From the present trial, we have previously reported similar glycaemic regulation as well as significant reductions in conventional independent predictors of CVD related to body weight, insulinaemia and cholesterolaemia during metformin than repaglinide treatments $(33,44)$. Our present findings extend these observations to include a favourable effect of metformin versus repaglinide on several independent predictors of CVD related to inflammation and endothelial dysfunction (TNF- $\alpha$, PAI-1-ag, t-PA-ag, sICAM-1 and vWf) (4, 6-8). These findings support the conclusion from the UK Prospective Diabetes Study (UKPDS) that the cardiovascular benefits of metformin in T2DM add beyond its effect on glycaemia (30). Most importantly, the present data indicate that this notion extends to include even non-obese patients with T2DM.

In predominantly obese patients with T2DM, metformin has previously been shown to decrease levels of vWf, sVCAM-1, ADMA, methylglyoxal (i.e. a major precursor in the formation of AGE) and albuminuria (19, 37, 45-48) and either decrease $(20,22,37$, 49-51) or having no significant effect $(19,20,24,37$, 50 ) on circulating levels of CRP, TNF- $\alpha$, PAI-1-ag, t-PA-ag, sICAM-1 and sE-selectin. For albuminuria, also an increase has been observed after metformin therapy (37). The observed lack of significant difference in the present study in the levels of IL-6, fibrinogen and adiponectin is in accordance with previous data for metformin in predominantly obese patients with T2DM $(20,24,26,37,48,51)$. Repaglinide has been shown to decrease levels of CRP, IL-6 and PAI-1-ag (21-23, 27) and either to decrease (27) or have no significant effect on levels of fibrinogen $(21,52)$ in predominantly obese patients with T2DM. The disparity between these results and our present data could be due to differences between studies in trial designs, e.g. observational versus intervention, uncontrolled versus controlled; patient characteristics, e.g. obese versus non-obese, OHA-naïve versus insulin-naïve patients etc. In fact, several CVD biomarkers (e.g. TNF- $\alpha$, adhesion molecules, vWf, t-PA-ag and PAI-1-ag) have been associated with phenotypes of the metabolic syndrome (16-18). From the present study, we have previously reported a non-significant difference in levels of $\mathrm{HbA}_{1 \mathrm{c}}$ (0.17 percentage points), significantly lower body weight $(1.58 \mathrm{~kg})$ and significantly lower fasting serum insulin levels $(13 \%)$ during metformin than repaglinide treatments (33). Accordingly, adjustment for the current levels of metabolic variables (i.e. $\mathrm{HbA}_{1 \mathrm{c}}$, body weight and insulin levels) influenced the study-drug effect on a number of the investigated CVD biomarkers (TNF- $\alpha$, t-PA-ag, sVCAM-1, sE-selectin and Amadori albumin). Levels of sVCAM-1, a marker of endothelial dysfunction, cardiovascular risk and mortality $(9,13)$, became significantly lower during metformin versus repaglinide, whereas levels of TNF- $\alpha$, t-PA-ag, sE-selectin and Amadori albumin were no longer significantly different between treatments.
Hence, even after adjustment for the potential effect of differences in the prevailing levels of metabolic variables, metformin treatment was still associated with significantly lower levels of several CVD biomarkers related to endothelial dysfunction (i.e. PAI-1-ag, vWf, sICAM-1 and sVCAM-1). This underscores the nonglycaemic as well as the non-obesity dependent potential beneficial effect of metformin treatment on CVD risk factors compared with an insulin secretagogue, repaglinide.

Despite our finding that hsCRP was not significantly different between treatments, the significantly lower levels of TNF- $\alpha$ during metformin suggest an increased anti-inflammatory effect with metformin versus repaglinide - although not significantly different after adjustment for metabolic variables as outlined. To our knowledge, the nature of causality between low-grade inflammation and metabolic variables has not been fully elucidated. Hence, whether changes in inflammatory markers (or the inflammatory state per se) are upstream or downstream (or both) from changes in metabolic variables remains to be established. We therefore conclude that the observed effect on TNF- $\alpha$ of metformin versus repaglinide was associated with changes in metabolic variables, but the causality between these changes cannot be determined from our data.

Recent in vitro studies have suggested that metformin activates the intracellular 'fuel-sensor', i.e. the AMPactivated kinase (AMPK). The activated AMPK, in turn, inhibits the TNF- $\alpha$-induced activation of the nuclear transcription factor, NF- $\kappa \mathrm{B}$, and hereby the NF- $\kappa \mathrm{B}-$ induced gene expression of sICAM-1, sVCAM and sE-selectin $(53,54)$. Hence, the observed decrease in several CVD biomarkers, in the present study, may be explained by actions of metformin via the pathway of AMPK, TNF- $\alpha$ and NF- $\kappa$ B. The changes from first-period baseline in the levels of $\mathrm{TNF}-\alpha$ were not significantly different from zero during either of the two treatments. Therefore, besides AMPK other, yet unknown, mechanisms (promoting, inhibitory and/or feedback) might contribute to the effect of metformin as well as repaglinide on CVD biomarkers. This is also underscored by the attenuating effect of metabolic variables on the treatment effects on several CVD biomarkers in the present study.

The lack of difference between interventions in blood pressure is in accordance with previous studies for both treatments in obese patients with T2DM $(23,24$, 55-58). The observed lower 24-h heart rate during repaglinide treatment, but similar 24-h blood pressure between treatment arms is in accordance with a previous study comparing glibenclamide with metformin (59). Otherwise, for both metformin $(24,60)$ and repaglinide $(52,58,61)$, the most previous studies have shown no significant difference in heart rate. Whether the increased heart rate with metformin will be of clinical importance is inconclusive from our data, but 
total mortality rises with increasing heart rate (62) and $\beta$-blocking agents reduces mortality in proportion to the degree of heart-rate reduction (63). However, the heart rate reduction in these trials has been at least double the magnitude (5-18 beats/min) compared with our findings.

The reduction in Amadori albumin with repaglinide suggests an improved glycaemic regulation during repaglinide versus metformin despite no significant difference in the levels of $\mathrm{HbA}_{1 \mathrm{c}}$ between treatments (33). Amadori albumin has been linked to the development of late-diabetic complications (14) and the lower levels hereof during repaglinide versus metformin suggest a potential clinical advantage during repaglinide treatment. However, other more late-stage glycation products, i.e. AGE peptides, CML or CEL (15) were not significantly different between treatments. Moreover, the between-treatment difference in Amadori albumin disappeared after correction for ambient levels of metabolic variables, including $\mathrm{HbA}_{1 \mathrm{c}}$.

The findings of changes in CVD biomarkers in favour of metformin (TNF- $\alpha$, PAI-1-ag, t-PA-ag, vWf, sICAM-1 and sE-selectin) as well as repaglinide (heart rate and Amadori albumin) suggest that the use of combination therapy might have beneficial potentials. Importantly, although the concept of combination therapy with metformin and insulin secretagogues is intriguing, it must be accepted only with great caution due to the increased mortality in patients who received such therapy in the UKPDS (30). However, recently Monami et al. published retrospective data suggesting that, even after adjusting for known confounders related to disease severity and co-morbidity, glibenclamide (the insulin secretagogue used in the UKPDS) in combination with metformin was associated with a higher mortality compared with repaglinide or other insulin secretagogues in combination with metformin therapy (64). The mechanism behind this finding was proposed to be an 100-fold higher binding to the sulphonylurea receptors with glibenclamide compared with repaglinide (65). We are unaware of previous studies reporting effects of combination therapy with metformin and repaglinide on CVD biomarkers. Currently, our group are analyzing data from a clinical trial including $\sim 120$ non-obese patients with T2DM who received combination therapy with metformin and repaglinide. Also, longer time (25 years) follow-up of UKPDS data should be available in near future. Such data will provide further insight into the issue of combination therapy of metformin and insulin secretagogues.

Although our findings are statistically significant, we cannot claim that they also possess unequivocally biological significant effects. Except for hsCRP and blood pressure $(66,67)$, which, in our present study, did not show significant differences between treatments, to our knowledge, intervention studies of hard CVD end points do not exist for other of the presented CVD biomarkers. In our present study, except for PAI-1-ag, showing a highly statistical significant $~ 25 \%$ betweentreatment difference, most other CVD biomarkers demonstrated lesser magnitudes of between-treatment differences in the range of $\sim 5-15 \%$. At first glance, such a magnitude of change might seem of little or no clinical relevance. However, clinical trial as well as epidemiological data, e.g. blood pressure and cholesterolaemia, indicate that changes in these variables within a magnitude of $\sim 5 \%$, or even less, can result in reductions in cardiovascular events (67-69). We therefore speculate that our findings of changes in CVD biomarkers of $\sim 5-25 \%$ might result in clinical relevant reductions in CVD.

In our present study, no patient presented with absolute insulin deficiency and only few patients were diagnosed with elevated GAD65 antibody titres or a MODY-3 genotype. Importantly, excluding such patients from the analysis made only minor or no substantial changes to the overall conclusions of the betweentreatment effects. Hence, we find our data to adequately represent subjects having a phenotype of non-obesity and T2DM according to available diagnostic tools in the everyday clinical practices (i.e. anthropometric measures, glycaemia and C-peptide determinations).

In conclusion, in non-obese patients with T2DM, metformin was more effective compared with repaglinide targeting several CVD biomarkers related to inflammation and endothelial dysfunction. However, repaglinide was slightly more effective compared with metformin targeting levels of Amadori albumin and heart rate. These treatment effects were achieved despite similar glycaemic regulation with both drugs and were independent of known CVD.

\section{Acknowledgements}

We would like to thank the following people and companies for their great help and support in carrying out this study: Novo Nordisk A/S (Bagsværd, Denmark) for financial support and study-medication supply; The Clinical Development Foundation at Steno Diabetes Center for financial support; Hexal A/S (GEA Ltd, Hvidovre, Denmark) for study-medication supply. Bente Blaaholm Nielsen as trial nurse; Birgitte Vilsbøl Hansen, Tina Ragnholm Juhl, Berit Ruud Jensen, Lotte Pietraszek, Ingelise Rossing and Lis Bülow as laboratory technicians. Novo Nordisk $\mathrm{A} / \mathrm{S}$ and the Clinical Development Foundation at Steno Diabetes Center co-sponsored the study financially. Novo Nordisk A/S supplied repaglinide and repaglinide-placebo tablets. Hexal A/S (GEA Ltd) supplied metformin and metformin-placebo tablets. The sponsors had no role in study design; collection, analysis or interpretation of data; writing the report; or the decision to submit the paper for publication. 


\section{Competing interests}

Søren Lund, Lise Tarnow, Merete Frandsen, Ulla Meng Smidt, Oluf Pedersen, Hans-Henrik Parving and Allan Vaag have reported equity in Novo Nordisk A/S. Lise Tarnow, Hans-Henrik Parving and Allan Vaag has received funds from Novo Nordisk A/S for research. Søren Lund and Allan Vaag have received fees from Novo Nordisk A/S for speaking and Allan Vaag has received fees from Novo Nordisk A/S for organizing education. Allan Vaag is a member of the editorial board for European Journal of Endocrinology. Søren Lund, Lise Tarnow, Merete Frandsen, Ulla Meng Smidt, Oluf Pedersen, Hans-Henrik Parving and Allan Vaag are present or former employees at Steno Diabetes Center, Gentofte, Denmark. Steno Diabetes Center is an independent academic institution owned by Novo Nordisk A/S and The Novo Nordisk Foundation.

\section{References}

1 Haffner SM, Lehto S, Rönnemaa T, Pyörälä K \& Laakso M. Mortality from coronary heart disease in subjects with type 2 diabetes and in nondiabetic subjects with and without prior myocardial infarction. New England Journal of Medicine 1998339 229-234.

2 Stamler J. Diabetes, other risk factors, and 12-yr cardiovascular mortality for men screened in the Multiple Risk Factor Intervention Trial. Diabetes Care 199316 434-444.

3 Beckman JA, Creager MA \& Libby P. Diabetes and atherosclerosis: epidemiology, pathophysiology, and management. Journal of the American Medical Association 2002287 2570-2581.

4 Thompson SG, Kienast J, Pyke SDM, Haverkate F \& van de Loo JCW. Hemostatic factors and the risk of myocardial infarction or sudden death in patients with angina pectoris. New England Journal of Medicine 1995332 635-641.

5 Ridker PM, Rifai N, Rose L, Buring JE \& Cook NR. Comparison of C-reactive protein and low-density lipoprotein cholesterol levels in the prediction of first cardiovascular events. New England Journal of Medicine 2002347 1557-1565.

6 Lapolla A, Piarulli F, Sartore G, Rossetti C, Martano L, Carraro P, De Paoli M \& Fedele D. Peripheral artery disease in type 2 diabetes: the role of fibrinolysis. Thrombosis and Haemostasis 2003 89 91-96.

7 Ridker PM, Rifai N, Pfeffer M, Sacks F, Lepage S \& Braunwald E. Elevation of tumor necrosis factor-alpha and increased risk of recurrent coronary events after myocardial infarction. Circulation $20001012149-2153$.

8 Tanne D, Haim M, Boyko V, Goldbourt U, Reshef T, Matetzky S, Adler Y, Mekori YA \& Behar S. Soluble intercellular adhesion molecule-1 and risk of future ischemic stroke: a nested casecontrol study from the bezafibrate infarction prevention (BIP) study cohort. Stroke 200233 2182-2186.

9 Blankenberg S, Rupprecht HJ, Bickel C, Peetz D, Hafner G, Tiret L \& Meyer J. Circulating cell adhesion molecules and death in patients with coronary artery disease. Circulation 2001104 1336-1342.

10 Schulze F, Lenzen H, Hanefeld C, Bartling A, Osterziel KJ Goudeva L, Schmidt-Lucke C, Kusus M, Maas R, Schwedhelm E, Strodter D, Simon BC, Mugge A, Daniel WG, Tillmanns H, Maisch B, Streichert T \& Boger RH. Asymmetric dimethylarginine is an independent risk factor for coronary heart disease: results from the multicenter coronary artery risk determination investigating the influence of ADMA concentration (CARDIAC) study. American Heart Journal 2006152 493-498.

11 Schulze MB, Shai I, Rimm EB, Li T, Rifai N \& Hu FB. Adiponectin and future coronary heart disease events among men with type 2 diabetes. Diabetes 200554 534-539.
12 Siroen MP, Teerlink T, Nijveldt RJ, Prins HA, Richir MC \& Van Leeuwen PA. The clinical significance of asymmetric dimethylarginine. Annual Review of Nutrition 200626 203-228.

13 Stehouwer CD, Gall MA, Twisk JW, Knudsen E, Emeis JJ \& Parving HH. Increased urinary albumin excretion, endothelial dysfunction, and chronic low-grade inflammation in type 2 diabetes: progressive, interrelated, and independently associated with risk of death. Diabetes 200251 1157-1165.

14 Cohen MP, Ziyadeh FN \& Chen S. Amadori-modified glycated serum proteins and accelerated atherosclerosis in diabetes: pathogenic and therapeutic implications. Journal of Laboratory and Clinical Medicine 2006147 211-219.

15 Smit AJ \& Lutgers HL. The clinical relevance of advanced glycation endproducts (AGE) and recent developments in pharmaceutics to reduce AGE accumulation. Current Medicinal Chemistry 200411 2767-2784.

16 Juhan-Vague I, Thompson SG \& Jespersen J. Involvement of the hemostatic system in the insulin resistance syndrome. A study of 1500 patients with angina pectoris. The ECAT Angina Pectoris Study Group. Arteriosclerosis and Thrombosis 199313 1865-1873.

17 Ryysy L \& Yki-Jarvinen H. Improvement of glycemic control by 1 year of insulin therapy leads to a sustained decrease in sE-selectin concentrations in type 2 diabetes. Diabetes Care 2001 24 549-554.

18 Katsuki A, Sumida Y, Murashima S, Murata K, Takarada Y, Ito K, Fujii M, Tsuchihashi K, Goto H, Nakatani K \& Yano Y. Serum levels of tumor necrosis factor-alpha are increased in obese patients with noninsulin-dependent diabetes mellitus. Journal of Clinical Endocrinology and Metabolism $1998 \mathbf{8 3} 859-862$.

19 Abbasi F, Chu JW, McLaughlin T, Lamendola C, Leary ET \& Reaven GM. Effect of metformin treatment on multiple cardiovascular disease risk factors in patients with type 2 diabetes mellitus. Metabolism 200453 159-164.

20 Chu NV, Kong AP, Kim DD, Armstrong D, Baxi S, Deutsch R, Caulfield M, Mudaliar SR, Reitz R, Henry RR \& Reaven PD. Differential effects of metformin and troglitazone on cardiovascular risk factors in patients with type 2 diabetes. Diabetes Care 200225 542-549.

21 Derosa G, Mugellini A, Ciccarelli L, Crescenzi G \& Fogari R. Comparison between repaglinide and glimepiride in patients with type 2 diabetes mellitus: a one-year, randomized, double-blind assessment of metabolic parameters and cardiovascular risk factors. Clinical Therapeutics 200325 472-484.

22 Derosa G, Mugellini A, Ciccarelli L, Crescenzi G \& Fogari R. Comparison of glycaemic control and cardiovascular risk profile in patients with type 2 diabetes during treatment with either repaglinide or metformin. Diabetes Research and Clinical Practice $200360161-169$.

23 Esposito K, Giugliano D, Nappo F \& Marfella R. Regression of carotid atherosclerosis by control of postprandial hyperglycemia in type 2 diabetes mellitus. Circulation 2004110 214-219.

24 Natali A, Baldeweg S, Toschi E, Capaldo B, Barbaro D, Gastaldelli A, Yudkin JS \& Ferrannini E. Vascular effects of improving metabolic control with metformin or rosiglitazone in type 2 diabetes. Diabetes Care 200427 1349-1357.

25 Yudkin JS, Panahloo A, Stehouwer C, Emeis JJ, Bulmer K, Mohamed-Ali V \& Denver AE. The influence of improved glycaemic control with insulin and sulphonylureas on acute phase and endothelial markers in type II diabetic subjects. Diabetologia 200043 1099-1106.

26 Phillips SA, Ciaraldi TP, Kong APS, Bandukwala R, Aroda V, Carter L, Baxi S, Mudaliar SR \& Henry RR. Modulation of circulating and adipose tissue adiponectin levels by antidiabetic therapy. Diabetes 200352 667-674.

27 Rizzo MR, Barbieri M, Grella R, Passariello N \& Paolisso G. Repaglinide has more beneficial effect on cardiovascular risk factors than glimepiride: data from meal-test study. Diabetes and Metabolism $200531255-260$.

28 Hundal RS \& Inzucchi SE. Metformin: new understandings, new uses. Drugs 200363 1879-1894. 
29 Inzucchi SE. Oral antihyperglycemic therapy for type 2 diabetes scientific review. Journal of the American Medical Association 2002 287 360-372.

30 UK Prospective Diabetes Study (UKPDS) Group. Effect of intensive blood-glucose control with metformin on complications in overweight patients with type 2 diabetes (UKPDS 34). Lancet $1998352854-865$.

31 Manson JE, Colditz GA, Stampfer MJ, Willett WC, Krolewski A, Rosner B, Arky RA, Speizer FE \& Hennekens CH. A prospective study of maturity-onset diabetes mellitus and risk of coronary heart disease and stroke in women. Archives of Internal Medicine $19911511141-1148$.

32 Adlerberth AM, Rosengren A \& Wilhelmsen L. Diabetes and longterm risk of mortality from coronary and other causes in middleaged Swedish men. A general population study. Diabetes Care 1998 21 539-545.

33 Lund SS, Tarnow L, Stehouwer CD, Schalkwijk CG, Frandsen M, Smidt UM, Pedersen O, Parving $\mathrm{HH}$ \& Vaag A. Targeting hyperglycaemia with either metformin or repaglinide in nonobese patients with type 2 diabetes: results from a randomized crossover trial. Diabetes, Obesity and Metabolism 20079 394-407.

34 Culy CR \& Jarvis B. Repaglinide: a review of its therapeutic use in type 2 diabetes mellitus. Drugs 200161 1625-1660.

35 Manzella D, Grella R, Abbatecola AM \& Paolisso G. Repaglinide administration improves brachial reactivity in type 2 diabetic patients. Diabetes Care 200528 366-371.

36 Schalkwijk CG, Ligtvoet N, Twaalfhoven H, Jager A, Blaauwgeers HG, Schlingemann RO, Tarnow L, Parving HH, Stehouwer CD \& vanHinsbergh VW. Amadori albumin in type 1 diabetic patients: correlation with markers of endothelial function, association with diabetic nephropathy, and localization in retinal capillaries. Diabetes 199948 2446-2453.

37 De Jager J, Kooy A, Lehert P, Bets D, Wulffele MG, Teerlink T, Scheffer PG, Schalkwijk CG, Donker AJ \& Stehouwer CD. Effects of short-term treatment with metformin on markers of endothelial function and inflammatory activity in type 2 diabetes mellitus: a randomized, placebo-controlled trial. Journal of Internal Medicine 2005257 100-109.

38 Teerlink T, Nijveldt RJ, de Jong S \& Van Leeuwen PA. Determination of arginine, asymmetric dimethylarginine, and symmetric dimethylarginine in human plasma and other biological samples by highperformance liquid chromatography. Analytical Biochemistry 2002 303 131-137.

39 Wrobel K, Wrobel K, Garay-Sevilla ME, Nava LE \& Malacara JM. Novel analytical approach to monitoring advanced glycosylation end products in human serum with on-line spectrophotometric and spectrofluorometric detection in a flow system. Clinical Chemistry 199743 1563-1569.

40 Teerlink T, Barto R, Ten-Brink HJ \& Schalkwijk CG. Measurement of Nepsilon-(carboxymethyl)lysine and Nepsilon-(carboxyethyl)lysine in human plasma protein by stable-isotope-dilution tandem mass spectrometry. Clinical Chemistry $2004501222-1228$.

41 Brown H \& Prescott R. Applied Mixed Models in Medicine, edn 2. West Sussex, UK: John Wiley \& Sons, Ltd, 2006.

42 Senn S. Cross-Over Trials in Clinical Research, edn 2. West Sussex, England: John Wiley \& Sons Ltd, 2002.

43 Senn S. Misunderstandings regarding clinical cross-over trials. Statistics in Medicine 200524 3675-3678.

44 Lund SS, Tarnow L, Frandsen M, Smidt UM, Pedersen O, Parving HH \& Vaag AA. Impact of metformin versus the prandial insulin secretagogue, repaglinide, on fasting and postprandial glucose and lipid responses in non-obese patients with type 2 diabetes. European Journal of Endocrinology 2008158 35-46.

45 Asagami T, Abbasi F, Stuelinger M, Lamendola C, McLaughlin T, Cooke JP, Reaven GM \& Tsao PS. Metformin treatment lowers asymmetric dimethylarginine concentrations in patients with type 2 diabetes. Metabolism $200251843-846$.

46 Beisswenger PJ, Howell SK, Touchette AD, Lal S \& Szwergold BS. Metformin reduces systemic methylglyoxal levels in type 2 diabetes. Diabetes 199948 198-202.
47 Amador-Licona N, Guizar-Mendoza J, Vargas E, Sanchez-Camargo G \& Zamora-Mata L. The short-term effect of a switch from glibenclamide to metformin on blood pressure and microalbuminuria in patients with type 2 diabetes mellitus. Archives of Medical Research 200031 571-575.

48 Nagi DK \& Yudkin JS. Effects of metformin on insulin resistance, risk factors for cardiovascular disease, and plasminogen activator inhibitor in NIDDM subjects. A study of two ethnic groups. Diabetes Care 199316 621-629.

49 Grant PJ. The effects of high- and medium-dose metformin therapy on cardiovascular risk factors in patients with type II diabetes. Diabetes Care $19961964-66$.

50 Testa R, Bonfigli AR, Piantanelli L, Manfrini S, Testa I \& Gregorio F. Relationship between plasminogen activator inhibitor type-1 plasma levels and the lipoprotein(a) concentrations in noninsulin-dependent diabetes mellitus. Diabetes Research and Clinical Practice 199633 111-118.

51 Grant PJ, Stickland MH, Booth NA \& Prentice CR. Metformin causes a reduction in basal and post-venous occlusion plasminogen activator inhibitor- 1 in type 2 diabetic patients. Diabetic Medicine 19918 361-365.

52 Marbury T, Huang WC, Strange P \& Lebovitz H. Repaglinide versus glyburide: a one-year comparison trial. Diabetes Research and Clinical Practice 199943 155-166.

53 Hattori Y, Suzuki K, Hattori S \& Kasai K. Metformin inhibits cytokine-induced nuclear factor kappaB activation via AMPactivated protein kinase activation in vascular endothelial cells. Hypertension $2006 \mathbf{4 7} 1183-1188$.

54 Isoda K, Young JL, Zirlik A, MacFarlane LA, Tsuboi N, Gerdes N, Schönbeck U \& Libby P. Metformin inhibits proinflammatory responses and nuclear factor- $\kappa \mathrm{B}$ in human vascular wall cells. Arteriosclerosis, Thrombosis, and Vascular Biology 200626 611-617.

55 Wulffele MG, Kooy A, de Zeeuw D, Stehouwer CD \& Gansevoort RT. The effect of metformin on blood pressure, plasma cholesterol and triglycerides in type 2 diabetes mellitus: a systematic review. Journal of Internal Medicine 2004256 1-14.

56 Uehara MH, Kohlmann NEB, Zanella MT \& Ferreira SRG. Metabolic and haemodynamic effects of metformin in patients with type 2 diabetes mellitus and hypertension. Diabetes, Obesity and Metabolism 20013 319-325.

57 Wulffele MG, Kooy A, Lehert P, Betst D, Donker AJM \& Stehouwer CDA. Does metformin decrease blood pressure in patients with type 2 diabetes intensively treated with insulin? Diabetic Medicine 200522 907-913.

58 Wolffenbuttel BH, Nijst L, Sels JP, Menheere PP, Muller PG \& Kruseman AC. Effects of a new oral hypoglycaemic agent, repaglinide, on metabolic control in sulphonylurea-treated patients with NIDDM. European Journal of Clinical Pharmacology $199345113-116$.

59 Sundaresan P, Lykos D, Daher A, Diamond T, Morris R \& Howes LG. Comparative effects of glibenclamide and metformin on ambulatory blood pressure and cardiovascular reactivity in NIDDM. Diabetes Care 199720 692-697.

60 Campbell IW, Duncan C, Patton NW, Broadhead T, Tucker GT \& Woods HF. The effect of metformin on glycaemic control, intermediary metabolism and blood pressure in non-insulindependent diabetes mellitus. Diabetic Medicine 19874 337-341.

61 Madsbad S, Kilhovd B, Lager I, Mustajoki P \& Dejgaard A. Comparison between repaglinide and glipizide in type 2 diabetes mellitus: a 1-year multicentre study. Diabetic Medicine 200118 395-401.

62 Thaulow E \& Eriksen JE. How important is heart rate? Journal of Hypertension 19919 (Suppl 7) S27-S30.

63 Kjekshus JK. Importance of heart rate in determining betablocker efficacy in acute and long-term acute myocardial infarction intervention trials. American Journal of Cardiology 198657 43F-49F.

64 Monami M, Luzzi C, Lamanna C, Chiasserini V, Addante F, Desideri CM, Masotti G, Marchionni N \& Mannucci E. Three-year mortality in diabetic patients treated with different combinations of insulin secretagogues and metformin. Diabetes/Metabolism Research and Reviews 200622 477-482. 
65 Tayek JA. Don't throw the baby out with the bath water: mortality with combination metformin and sulfonylurea. Diabetes/Metabolism Research and Reviews 200723 85-86.

66 Ridker PM, Cannon CP, Morrow D, Rifai N, Rose LM, McCabe CH, Pfeffer MA \& Braunwald E. C-reactive protein levels and outcomes after statin therapy. New England Journal of Medicine 2005352 20-28.

67 Patel A, MacMahon S, Chalmers J, Neal B, Woodward M, Billot L, Harrap S, Poulter N, Marre M, Cooper M, Glasziou P, Grobbee DE, Hamet P, Heller S, Liu LS, Mancia G, Mogensen CE, Pan CY, Rodgers A \& Williams B. Effects of a fixed combination of perindopri and indapamide on macrovascular and microvascular outcomes in patients with type 2 diabetes mellitus (the ADVANCE trial): a randomised controlled trial. Lancet 2007 370 829-840.
68 Johnson CL, Rifkind BM, Sempos CT, Carroll MD, Bachorik PS, Briefel RR, Gordon DJ, Burt VL, Brown CD, Lippel K \& Cleeman JI. Declining serum total cholesterol levels among US adults. The National Health and Nutrition Examination Surveys. Journal of the American Medical Association $19932693002-3008$.

69 Gillum RF. Trends in acute myocardial infarction and coronary heart disease death in the United States. Journal of the American College of Cardiology 199423 1273-1277.

Received 28 January 2008

Accepted 5 February 2008 